\title{
Joint Coverage Enhancement by Power Allocation in Poisson Clustered Out-of-Band D2D Networks
}

\author{
Jinchuan Tang, Student Member, IEEE, Gaojie Chen, Member, IEEE, and Justin P. Coon, Senior Member, IEEE
}

\begin{abstract}
In this paper, we consider a Poisson clustered outof-band device-to-device (D2D) network operating in Rayleigh fading channels and propose two optimization schemes based on the joint coverage probability to allocate the transmit power to D2D user equipments (DUEs) in both the target cluster and the whole network. The joint coverage probability accounts for the spatial correlation of the interference among the DUEs. With a differing amount of location information being available among the DUEs in centralized and localized communication solutions, the joint coverage probability is formulated based on the coherent, non-coherent, and single-cluster approximated pairwise coverage probabilities. By maximizing the joint coverage probability formulated in this way, the robustness of the network can be enhanced. Meanwhile, to fulfill the green aspect of future 5G communications, the battery life of the DUEs will be prolonged by minimizing the power consumption for a given joint coverage probability requirement. The formulations of the two optimization problems above are not convex but are transformed to convex by using geometric programming. The simulation results show that, compared with the conventional LTE open loop and fixed power schemes, the joint coverage probability and power consumption are respectively optimized by applying the proposed schemes.
\end{abstract}

Index Terms-Poisson clustered, out-of-band D2D, joint coverage probability, power allocation, convex optimization.

\section{INTRODUCTION}

A $\mathrm{S}$ introduced in the Release 12 by the 3rd Generation Partnership Project (3GPP), device-to-device (D2D) communications can provide proximity based services (ProSe) for mission-critical applications in long-term evolution (LTE) [1]. It allows the mobile devices in proximity to communicate directly without the intermediate transmission to an evolved Node B (eNB) and has been explored by researchers to support future 5G communications [2]. In out-of-band D2D communications, D2D and cellular communications can be established simultaneously in two different frequency bands with potential technologies such as $\mathrm{WiFi}$, ZigBee, Bluetooth and LTE-Unlicensed [3]. Based on the difference in controlled categories, out-of-band D2D communications can be further divided into controlled and autonomous solutions. The former uses cellular technologies to manage the establishment of D2D

Copyright (c) 2015 IEEE. Personal use of this material is permitted. However, permission to use this material for any other purposes must be obtained from the IEEE by sending a request to pubs-permissions@ieee.org

This work was supported by EPSRC through "Spatially Embedded Networks" under Grant EP/N002350/1 and through M3NETs under Grant EP/R006377/1.

J. Tang and J. P. Coon are with the Department of Engineering Science, Parks Road, University of Oxford, Oxford, UK, OX1 3PJ, Emails: \{jinchuan.tang and justin.coon\}@eng.ox.ac.uk.

G. Chen is with the Department of Engineering, University of Leicester, Leicester, UK, LE1 7RH, Email: gaojie.chen@leicester.ac.uk. links in a centralized manner, while the latter allows the terminals to control the D2D interfaces in a localized manner to reduce overhead to the cellular network [4]. Early studies have shown that D2D communications can improve data rate, increase spectral efficiency, reduce power consumption, and further offload the traffic in dense mobile communication networks [5], [6]. Enabling D2D networks would benefit not only mission-critical communications such as public safety communications [7], but also commercial D2D communications for future 5G systems [8], [9].

Apart from these promising benefits, achieving reliable connections is still challenging in D2D networks. According to IMT-2020, the capability to support maximum connection density of $10^{6} / \mathrm{km}^{2}$ is to be expected in future communication networks [10]. However, the limited number of resource blocks (RBs) and scheduling policies in current LTE systems may not fulfill such a requirement. In the networks where the density is high, and energy or spectrum efficiency is of utmost importance, the clustering of the DUEs appears to be a good option, because it can bring lower signaling overhead, higher spectral efficiency, and better energy efficiency compared with legacy cellular system [11]. Meanwhile, due to the fact that the locations of DUEs are constrained by factors such as geographical differences, urban planning, and land availability, the D2D communications could also inevitably be clustered and mutually interfer in the existed networks [3], [11]-[13]. The clustered network models which proceed within such a paradigm for D2D content distribution can be found in [14][17].

To model and analyze the performance of the clustered networks, a class of Poisson cluster processes (PCPs) has been used. The performance analyses of a random network are given mainly by the fact that the connection reliability for a DUE pair could be severely affected by the interference due to the concurrent transmitting user equipments (UEs) on the same channel [13]. The authors of [18] analyzed the outage probability of the modified Thomas cluster based LTE two-tier femtocell networks, where all UEs transmit with a fixed power. The authors of [19] studied the area spectral efficiency of the modified Thomas clusters for the clustered D2D networks. However, interference observed at a given location is temporally and/or spatially correlated, ignoring this correlation by only considering the coverage probability of a typical link may lead to the incorrect characterization of network performance [20]. To identify such correlation, the joint coverage probability, namely joint complementary cumulative distribution function (CCDF) of SINR, has been used to study the joint statistics across space or time [21]-[23]. 
These analyses are normally built on the assumption that all UEs have either the fixed power or path loss inversion power strategy, which does not guarantee the optimum or desired joint coverage performance.

Power allocation is an effective approach to interference management to reducing interference and enhancing the performance of a wireless network. Traditional power control schemes for LTE are focusing on reducing the interference between D2D and cellular users in the inband D2D communications [24]. For example, in [25] and [26], the Poisson based analytical modeling and optimization for spectrum underlay D2D communications were respectively studied. A strategy to use the harvested RF energy from interferers to perform path loss inversion power control for D2D communications was considered in [27]. The centralized and localized power allocation methods for Poisson based D2D underlaid cellular networks were investigated in [28]. Regardless of these achievements for inband D2D communications, how to allocate power in a clustered out-of-band D2D network for achieving the optimum joint coverage performance is still an open topic. For the autonomous out-of-band solution, due to the absence of a central controller, performing power control on a link-by-link basis in a large-scale wireless network is a complicated task. The common transmit power level has been used as a viable method for the out-of-band D2D networks [13], [19], [29]-[31]. Meanwhile, to the best knowledge of the authors, interference management techniques are lacking for the controlled D2D out-of-band solution, and traditional interference management such as the case in [24] was focusing on reducing the interference between D2D and cellular users in the inband D2D communications based on techniques such as power allocation. Furthermore, for a guaranteed joint coverage performance, the idea of green radio communications requires the action to further reduce power consumption in order to limit the subsequent impact on the environment in the form of $\mathrm{CO}_{2}$ emissions [32].

In this paper, therefore, we propose two power allocation frameworks for performance optimizations in the target cluster and the whole network in the Poisson clustered out-of-band D2D networks. The simulations have shown that the performance of the target cluster and the whole network given by the proposed schemes will be optimum over the fixed power and open loop schemes.

The contributions of the paper are:

1) We propose two optimization frameworks to enhance coverage performance in the target cluster and the whole network. They are suitable for both commercial content distributions and the public safety applications where high joint coverage probability and lower power consumption are desired.

2) We formulate the joint coverage probability based on the coherent, non-coherent lower bound, and single-cluster approximation coverage probabilities, which depends on a differing amount of location information of the clustered DUEs.

3) We prove a lower bound of the inter-cluster interference from Matérn clusters based on its conditional distance probability density function (PDF), and proved the convex optimization problems.

The rest of the paper is structured as follows. Section II begins with the system model and addresses three different formulations for the pairwise coverage probabilities. Section III focuses on the formulation and optimizations on the joint coverage probability. Section IV gives the simulation results and discussion, and Section $\mathrm{V}$ concludes the paper.

\section{SySTEM MODEL}

We consider a stochastic model for the clustered out-ofband D2D networks, where the gathering behavior of DUEs is modeled by the PCPs. For a given channel resource, each cluster is assumed to contain an average of $\bar{m}$ D2D pairs. For each pair, there is one content requester (receiving DUE) and one content provider (transmitting DUE). Two types of PCPs, i.e., Matérn and modified Thomas cluster processes, in Fig. 1 are investigated. The origins of the clusters are distributed in the plane according to a Poisson point process $\Phi_{o}$ with intensity $\lambda_{o}$. For an origin at $\mathbf{x}_{o}$, the Matérn clustered DUEs are independently, identically and uniformly distributed in the disc of radius $R$. For the same origin, the modified Thomas clustered DUEs obey the isotropic Gaussian distribution $\mathcal{N}\left(\mathbf{x}_{o}, \sigma^{2} \mathbf{I}\right)$ with standard deviation $\sigma$ along each coordinate axis and $\mathbf{I}$ is a $2 \times 2$ identity matrix [33]. We investigate the optimization frameworks on the DUEs, which share the same channel resources throughout the clusters. The reuse of channel resources within a cluster is also allowed to avoid the enormous signaling overhead caused by accommodating feedback from the receivers, as long as the system performance is acceptable [34], [35]. The conditional density functions of DUEs are given by [13]

a) Matérn:

$$
f\left(\mathbf{u} \mid \mathbf{x}_{o}, R\right)=\frac{1}{\pi R^{2}}, \text { for } r=\left\|\mathbf{u}-\mathbf{x}_{o}\right\| \leq R ;
$$

b) Modified Thomas:

$$
f\left(\mathbf{u} \mid \mathbf{x}_{o}, \sigma^{2}\right)=-\frac{1}{2 \pi \sigma^{2}} \exp \left(-\frac{\left\|\mathbf{u}-\mathbf{x}_{o}\right\|^{2}}{2 \sigma^{2}}\right) .
$$

Since the probability that a Gaussian random variable $X \sim$ $\mathcal{N}\left(0, \sigma^{2}\right)$ lies in the interval $[-3 \sigma, 3 \sigma]$ is equal to 0.997 [36], we let $\sigma=R / 3$ so that the average numbers of the Matern and the modified Thomas clustered DUEs within radius $R$ are approximately the same. Under this condition, we can compare the performance difference between the uniformly distributed and the normally concentrated DUEs. Without loss of generality, we assume that the channels are static over a block time period and independently and identically Rayleigh distributed from one block to the next [37].

\section{A. Received signal}

For simplicity of notation, we give unique node indices $\{i\}$ to all DUEs. A node $i$ has a transmit power $P_{i}$, and the received signal power at node $j$ while transmitting from node $i$ is [38]

$$
P_{i j}=G_{e} \ell\left(d_{i j}\right) F_{i j} P_{i}
$$




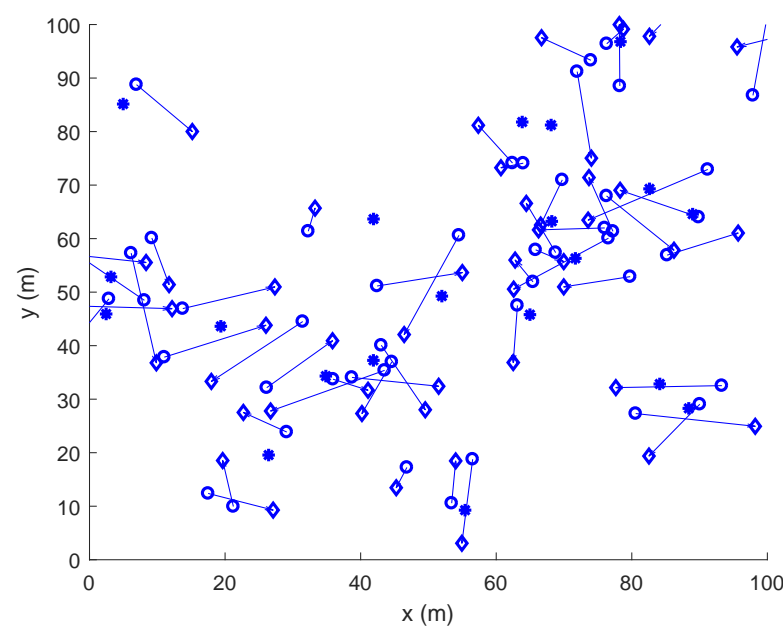

(a) Matérn clusters, $R=12.5 \mathrm{~m}$.

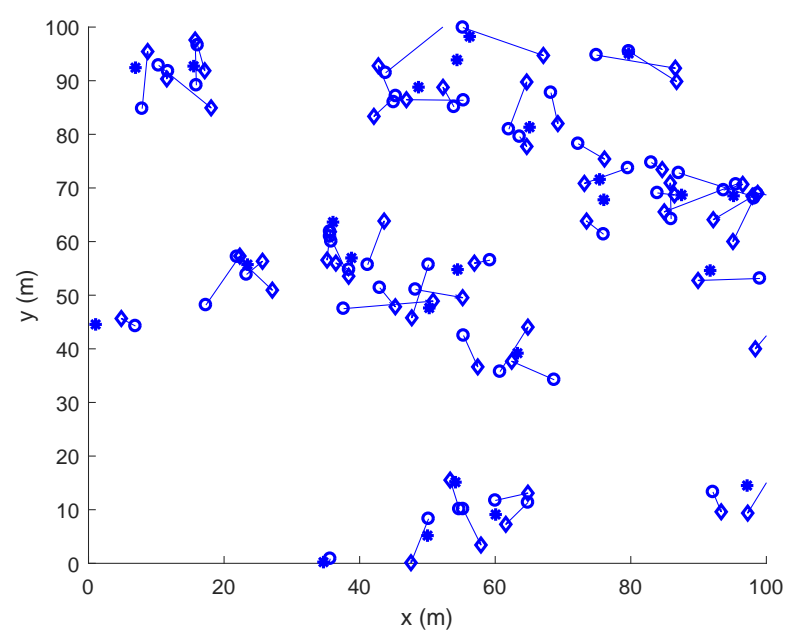

(b) Modified Thomas clusters, $\sigma \approx 4.2 \mathrm{~m}$.

Fig. 1: Illustration of clustered transmitting and receiving DUEs. $*$ : cluster origins, $\circ$ : transmitting DUEs, $\diamond$ : receiving DUEs, $\rightarrow$ : transmission link, $\lambda_{o}=2000$ clusters $/ \mathrm{km}^{2}, \bar{m}=2$, and the connection density is $4 \times 10^{3} / \mathrm{km}^{2}$.

where $\ell\left(d_{i j}\right)=(\lambda / 4 \pi)^{2} d_{i j}^{-\alpha} ; \lambda$ represents the carrier wavelength; $d_{i j}$ is the pair distance between node $i$ and $j ; \alpha$ is the path loss exponent. Although this model has a singularity as $d_{i j} \rightarrow 0$, such a singularity has a negligible effect on the results from a coverage perspective [39], [40]. We assume that the gain due to coding, transmitting antenna and receiving antenna at any receiving DUE is $G_{e} . F_{i j}$ models the power of Rayleigh fading which obeys the exponential distribution with unit mean [41]. Due to lack of interference management in the existing models for out-of-band networks, we also assume that the transmit power $P_{j}$ for any DUE without applying power schemes are fixed to be a value denoted by the common transmit power $P_{d}$.

\section{B. Signal to interference-plus-noise ratio}

For a given realization of the cluster process, let $N_{c}$ denote the number of clusters and $L_{n}$ represent the set of indices belonging to the transmitting DUEs inside cluster $n \in\left(1, \ldots, N_{c}\right)$, the union of all simultaneously transmitting DUE sets is denoted by $L_{T}=\bigcup_{n} L_{n}$. In the presence of both intra-cluster and inter-cluster interference, the signal to interference-plus-noise ratio (SINR) at node $j$ while receiving a signal from node $i$ is given by [42]

$$
\operatorname{SINR}_{i j}=\frac{P_{i j}}{N_{0}+I_{i j}},
$$

where $N_{0}$ is the additive white Gaussian noise (AWGN) power at each node. The interference at node $j$ while receiving the signal from node $i$ is written as

$$
I_{i j}=\sum_{k \in L_{T} \backslash\{i\}} P_{k j},
$$

where $L_{T} \backslash\{i\}$ denotes the set of all the simultaneously transmitting DUEs from all of the clusters.

\section{Coverage probability}

In this section, we formulate three types of coverage probabilities named coherent, non-coherent lower bound, and single-cluster approximation coverage probabilities. The major difference between the coherent and non-coherent coverage probabilities $^{1}$ is that the former requires all location information of the DUEs to be reported back to a central controller such as the base station, while the latter requires the DUEs within the target cluster to report their location information to either the centralized controller or a DUE member working as the localized controller. Besides, the non-coherent coverage probability also requires the statistical information on the distribution of the interference of neighboring clusters. This statistical information can be obtained at the centralized controller by analyzing the received location information of inter-cluster DUEs in the long term. It will be further sent to the localized controller if the controller is in charge of performing the optimization. The single-cluster approximation considers only the intra-cluster interference, which requires the location information of the DUEs within the same cluster to be sent to either the centralized or the localized controller.

1) Coherent coverage probability: The coverage probability of a link is the probability that the SINR of the link is higher than a predetermined threshold $\beta=2^{t}-1$ with link spectrum efficiency $t \mathrm{bit} / \mathrm{s} / \mathrm{Hz}$. Given the distances and transmit powers from all transmitting nodes to a receiving node $j$, the coherent coverage probability from nodes $i$ to $j$ in a cluster is written as

$$
\begin{aligned}
p_{i j}^{c} & =\mathbb{P}\left\{\operatorname{SINR}_{i j}>\beta\right\}=\mathbb{P}\left\{F_{i j}>\frac{\beta\left(N_{0}+I_{i j}\right)}{G_{e} \ell\left(d_{i j}\right) P_{i}}\right\} \\
& =\exp \left(-\frac{\beta N_{0}}{G_{e} \ell\left(d_{i j}\right) P_{i}}\right) \prod_{k \in L_{T} \backslash\{i\}}\left(1+\frac{\beta \ell\left(d_{k j}\right) P_{k}}{\ell\left(d_{i j}\right) P_{i}}\right)^{-1},
\end{aligned}
$$

\footnotetext{
${ }^{1}$ The use of the terms "coherent" and "non-coherent" is inspired by 1) joint coherent and non-coherent transmission where spatial channel state information feedback is only needed for the former; 2) coherent and noncoherent detection where the knowledge of the phase of the carrier frequency is needed for the former.
} 
where $\mathbb{P}\{\cdot\}$ denotes the probability of an event. The analysis above needs to have the knowledge of distances and transmit powers from all the concurrent transmitting nodes to a receiving node which could result in heavy traffic on the network back-haul [43].

2) Non-coherent coverage probability: In some scenarios, where only the knowledge of the location information of the DUEs within the target cluster is known, and the transmit power of inter-clusters is fixed due to lack of interference management, the statistical properties of the clusters can be exploited to formulate a non-coherent coverage probability. Given the distances and transmit powers from all the transmitting nodes to a receiving node inside the same cluster and the cluster statistics, the non-coherent coverage probability between the transmitting node $i$ and receiving node $j$ in cluster $n$ is written as

$$
\begin{aligned}
p_{i j}^{n c}= & \exp \left(-\frac{\beta N_{0}}{G_{e} \ell\left(d_{i j}\right) P_{i}}\right) \prod_{k \in L_{n} \backslash\{i\}}\left(1+\frac{\beta \ell\left(d_{k j}\right) P_{k}}{\ell\left(d_{i j}\right) P_{i}}\right)^{-1} \\
& \times \mathcal{L}_{I}\left(\frac{\beta}{G_{e} \ell\left(d_{i j}\right) P_{i}}\right),
\end{aligned}
$$

where $\mathcal{L}_{I}(s)$ is the Laplace transform of the inter-cluster interference given by the probability generating functional of the Neyman-Scott cluster process [13]. Hence, we have

$$
\mathcal{L}_{I}(s)=\exp \left(-2 \pi \lambda_{o} \int_{0}^{\infty}\left[1-\xi\left(s, R_{y}\right)\right] R_{y} \mathrm{~d} R_{y}\right),
$$

where

$$
\xi\left(s, R_{y}\right)=\exp \left\{-\bar{m} \int_{0}^{\infty} \frac{g(x)}{s^{-1}+g(x)} f_{R_{d}}\left(x \mid R_{y}\right) \mathrm{d} x\right\},
$$

and $g(x)=G_{e} P_{d} \ell(x)$. (8) is the result of the momentgenerating function of the Poisson process $\Phi_{o}$ for parent points. (9) is the Laplace transform of the interference from one cluster, given the distance $R_{y}$ between the origin of the interference cluster and the receiving node in cluster $n . P_{d}$ is the common transmit power of the DUEs in the network. $f_{R_{d}}\left(r_{d} \mid R_{y}\right)$ is the conditional distance PDF of DUEs. For Matérn and modified Thomas cluster processes, their conditional distance PDFs are respectively given by [35], [44]

a) Matérn:

$$
\begin{gathered}
f_{R_{d}}\left(r_{d} \mid R_{y}\right)=\frac{c\left(r_{d}\right)}{\pi R^{2}}, \\
c\left(r_{d}\right)= \begin{cases}2 \pi r_{d}, & r_{d} \leq R-R_{y} \\
2 \pi r_{d}-c_{1}\left(r_{d}\right), & R-R_{y}<r_{d} \text { and } r_{d}^{2} \leq R^{2}-R_{y}^{2} \\
c_{1}\left(r_{d}\right), & R^{2}-R_{y}^{2}<r_{d}^{2} \\
0, & \text { otherwise, }\end{cases}
\end{gathered}
$$

where $c_{1}\left(r_{d}\right)$ is defined by (12) on top of the next page.

b) Modified Thomas:

$$
f_{R_{d}}\left(r_{d} \mid R_{y}\right)=\frac{r_{d}}{\sigma^{2}} \exp \left(-\frac{r_{d}^{2}+R_{y}^{2}}{2 \sigma^{2}}\right) I_{0}\left(\frac{r_{d} R_{y}}{\sigma^{2}}\right)
$$

where $I_{0}(z)$ is the zeroth order modified Bessel function of the first kind.

Numerically evaluating the integral form of (8) is timeconsuming and does not lead to much insight. Thus, we propose a closed-form lower bound on the Laplace transform of the inter-cluster interference with the following Lemma.

Lemma 1. The Laplace transforms of the inter-cluster interference of both the Matern and modified Thomas cluster processes are lower bounded on

$$
\mathcal{L}_{I}(s) \geq \exp \left(-\pi \lambda_{o} \bar{m} \frac{\left(s G_{e}\left(\frac{\lambda}{4 \pi}\right)^{2} P_{d}\right)^{2 / \alpha}}{\operatorname{sinc}(2 / \alpha)}\right) .
$$

Proof: For a proof of this lemma for the Matérn cluster process based on the conditional distance PDF (10), please see Appendix A. For a proof of this lemma for the modified Thomas cluster process, please see [19].

As a result of Lemma 1, a lower bound of the non-coherent coverage probability is written as

$$
\begin{aligned}
p_{i j}^{n c} \geq & \exp \left(-\frac{\beta N_{0}}{G_{e} \ell\left(d_{i j}\right) P_{i}}\right) \prod_{k \in L_{n} \backslash\{i\}}\left(1+\frac{\beta \ell\left(d_{k j}\right) P_{k}}{\ell\left(d_{i j}\right) P_{i}}\right)^{-1} \\
& \times \exp \left(-\pi \lambda_{o} \bar{m} \frac{\left(\beta P_{d} /\left(d_{i j}^{-\alpha} P_{i}\right)\right)^{2 / \alpha}}{\operatorname{sinc}(2 / \alpha)}\right) .
\end{aligned}
$$

3) Single-cluster approximation coverage probability: In the cases where only the intra-cluster interference is taken into consideration, the single-cluster approximation coverage probability between the transmitting node $i$ and receiving node $j$ in cluster $n$ is given by

$p_{i j}^{s c}=\exp \left(-\frac{\beta N_{0}}{G_{e} \ell\left(d_{i j}\right) P_{i}}\right) \prod_{k \in L_{n} \backslash\{i\}}\left(1+\frac{\beta \ell\left(d_{k j}\right) P_{k}}{\ell\left(d_{i j}\right) P_{i}}\right)^{-1}$,

where the only difference between (6) and (16) is that $L_{n}$ is used instead of $L_{T}$. The relationship between non-coherent and single-cluster approximated is given by

$$
p_{i j}^{n c}=p_{i j}^{s c} \mathcal{L}_{I}\left(\frac{\beta}{G_{e} \ell\left(d_{i j}\right) P_{i}}\right) .
$$

\section{Joint Coverage Probability and Convex OPTIMIZATION}

In this section, we formulate the convex optimization problems to maximize joint coverage probability and minimize power consumption for the target cluster and the whole network.

\section{A. Joint coverage probability}

Let $\kappa$ denote either the target cluster or all clusters in the network for the optimization of either the target cluster or the whole network, the joint coverage probability of $\kappa$ is determined by the coverage probability of each DUE pair within $\kappa$, which can be formulated as

$$
p_{s}=\prod_{i \in L_{\kappa}} p_{i k_{i}}
$$




$$
c_{1}\left(r_{d}\right)= \begin{cases}2 r_{d} \arcsin \frac{\sqrt{4 R_{y}^{2} r_{d}^{2}-\left(R_{y}^{2}-R^{2}+r_{d}^{2}\right)^{2}}}{2 R_{y} r_{d}}, & \left(R_{y}-R+r_{d}\right)\left(R_{y}+R-r_{d}\right)\left(R_{y}-R-r_{d}\right) \leq 0 \\ 0, & \text { otherwise }\end{cases}
$$

where $k_{i}$ is the index of the corresponding receiving DUE that DUE $i$ connects to, and $p_{i k_{i}}$ is the coverage probability from $i$ to $k_{i}$.

The joint coverage probability of the target can be formulated based on either the coherent formulation in (6), non-coherent lower bound in (15) or single-cluster approximation in (16). Since the location information of all the nodes must be known in (6), the joint coverage probabilities based on the coherent formulation is only suitable for the centralized communication networks. Meanwhile, the joint coverage probabilities based on non-coherent lower bound require the knowledge of the location information of the DUEs within the target cluster and the statistical information on the distribution of inter-cluster interference. Thus, it is suitable for both the centralized and partially localized communication networks. Furthermore, the joint coverage probabilities based on single-cluster approximation require location information of the DUEs, which is suitable for both the centralized and localized communication networks.

Meanwhile, the joint coverage probability of the whole network can be formulated based on the coherent formulation and single-cluster approximation, but it is not feasible to use the non-coherent lower bound, because clusters could have different transmit power levels during optimizations, and the corresponding analytic expression for the lower bound would not exist. Like the target cluster case, the coherent formulation can be applied to the centralized communication networks. Meanwhile, since the coverage probabilities of the DUE pairs in one cluster are independent of another in single-cluster approximation, thus it can be applied to both the centralized and localized communication networks.

\section{B. Problem formulation and convex optimization}

1) Maximizing joint coverage probability: For many applications including content distribution and mission-critical communications, it is desirable that the joint coverage of the target cluster or the whole network is maximized. This is because that the D2D communications within the cluster or the network could have the best joint performance in operating concurrently towards a given link spectrum efficiency. Meanwhile, it is even better if a similar or lower amount of average power per DUE is consumed compared with other power allocation schemes. To achieve such a goal, we formulate the optimization problem to maximize the joint coverage probability of $\kappa$.

$$
\begin{array}{ll}
\text { maximize } & p_{s}\left(\mathbf{P}_{\kappa}\right) \\
\text { subject to } & \mathrm{P}_{\mathrm{L}} \leq P_{i} \leq \mathrm{P}_{\mathrm{U}}, \quad i \in L_{\kappa},
\end{array}
$$

with variables $\mathbf{P}_{\kappa}$, where $\mathbf{P}_{\kappa}=\left\{P_{i}\right\}$ is a vector that represents the transmit power levels for all the transmitting DUEs within $\kappa$, and the function $p_{s}\left(\mathbf{P}_{n}\right)$ explicitly denotes (18) as a

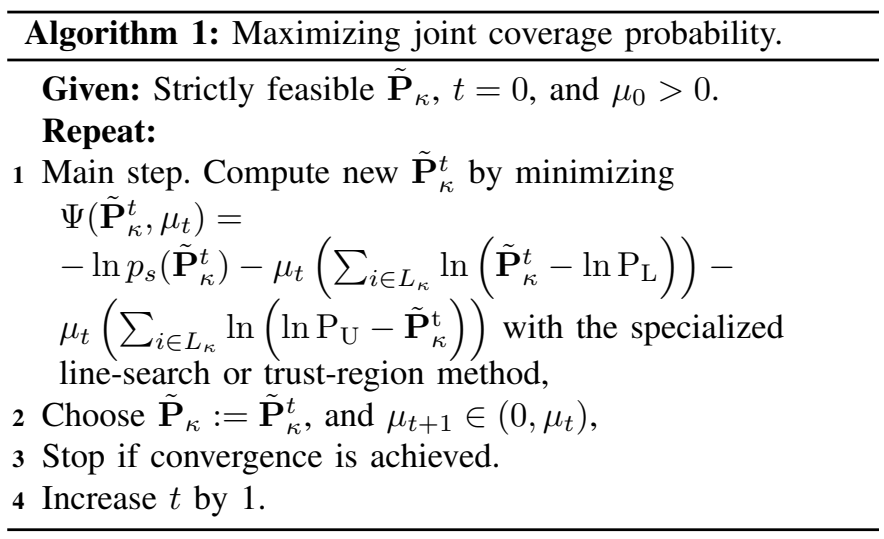

function of $\mathbf{P}_{n} ; \mathrm{P}_{\mathrm{L}}$ and $\mathrm{P}_{\mathrm{U}}$ are respectively the lower and upper bounds of the transmit power for DUEs which take part in the optimization; $\mathrm{P}_{\mathrm{L}}$ is given by the minimum transmit power ${ }^{2}$ of the DUE; $\mathrm{P}_{\mathrm{U}}$ is set as $P_{d}$, so that, when optimizing the target cluster, the performance boost after optimization won't raise at the cost of other interfering clusters with the fixed transmit power $P_{d}$. The optimization problem formulated above is not convex. To further analyze the optimization problem, we require the following theorem:

Theorem 1. The joint coverage probability in (19) are logconcave in $\tilde{\mathbf{P}}_{\kappa}$ when formulated based on the coherent formulation, non-coherent lower bound and single-cluster approximation, where the $i$ th element of $\tilde{\mathbf{P}}_{\kappa}$ is $\tilde{P}_{i}=\ln P_{i}$.

Proof: See Appendix B.

As a result of Theorem 1, the optimization problem is transformed into

$$
\begin{array}{ll}
\operatorname{maximize} & \ln p_{s}\left(\tilde{\mathbf{P}}_{\kappa}\right) \\
\text { subject to } & \ln \mathrm{P}_{\mathrm{L}} \leq \tilde{P}_{i} \leq \ln \mathrm{P}_{\mathrm{U}}, \quad i \in L_{\kappa},
\end{array}
$$

with variable $\tilde{\mathbf{P}}_{\kappa}$, which is a geometric programming format [47]. The surge of computational power, algorithms, and new coding approaches makes it possible to solve modest-size convex optimization problems in microsecond or millisecond scales [47], [48]. Hence, the computational complexity of the power allocation solution given in (21) would not pose a difficulty in practice. The preliminary algorithm based on interior-point methods [49] is summarized in Algorithm 1, and an interior-point solver to (21) - (22), which combines line search and trust region steps [50], is available in Matlab optimization toolbox.

2) Minimizing power consumption: In the green communications paradigm, reducing device transmit power consumption is beneficial to save the battery life of the DUEs and

\footnotetext{
${ }^{2}$ It is a design challenge to make a transmitter with a tunable transmit power range under certain low power level [45]. Therefore, the minimum transmit power for a UE is given by $P_{L}$ instead of 0 Watt. Please see Ch. 6 of [46] for minimum UE transmit power in LTE networks.
} 


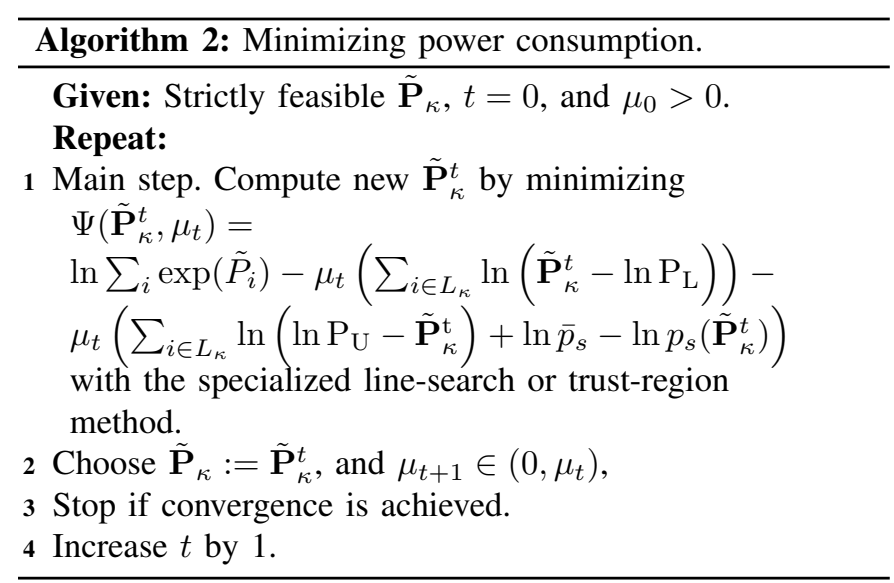

the subsequent impact on the environment in the form of $\mathrm{CO}_{2}$ emissions. If an acceptable joint coverage probability is provided, how to minimize transmit power consumption to achieve such a joint coverage probability has been raised. To tackle it, we provide the following formulas to minimize the total transmit power consumption:

$$
\begin{array}{ll}
\operatorname{minimize} & \sum_{i} P_{i} \\
\text { subject to } & \mathrm{P}_{\mathrm{L}} \leq P_{i} \leq \mathrm{P}_{\mathrm{U}}, \quad i \in L_{\kappa}, \\
& \bar{p}_{s}<p_{s}\left(\mathbf{P}_{\kappa}\right),
\end{array}
$$

with variables $\mathbf{P}_{\kappa}$, where $\bar{p}_{s}$ is a specified threshold corresponding to the minimum joint coverage probability for the DUE pairs within a cluster. Since (25) is not convex, we need the following theorem to transform the original optimization problem into a convex optimization problem.

Theorem 2. Let $\tilde{P}_{i}=\ln P_{i}$, (23) - (25) can be transformed into a convex optimization problem, which is given by

$$
\begin{array}{ll}
\text { minimize } & \ln \sum_{i} \exp \left(\tilde{P}_{i}\right) \\
\text { subject to } & \ln \mathrm{P}_{\mathrm{L}} \leq \tilde{P}_{i} \leq \ln \mathrm{P}_{\mathrm{U}}, \quad i \in L_{\kappa}, \\
& \ln \bar{p}_{s}-\ln p_{s}\left(\tilde{\mathbf{P}}_{\kappa}\right)<0,
\end{array}
$$

with variable $\tilde{\mathbf{P}}_{\kappa}$.

Proof: See Appendix C.

The corresponding preliminary algorithm based on interiorpoint methods is summarized in Algorithm 2, and (26) - (28) can also be implemented by using Matlab interior-point solver.

\section{Simulation Results And Discussion}

In order to test the proposed algorithms against relevant benchmarks, simulations based on LTE parameters were developed.

\section{A. Simulation configuration}

In the simulation, DUEs are randomly deployed on a plane with area $A$ according to Matérn and modified Thomas cluster processes. The parameters used in the simulation are given in Table I unless otherwise specified. The Matlab 'fmincon'
TABLE I: System parameters

\begin{tabular}{ll}
\hline Parameter & Value \\
\hline Layout & Random clusters \\
Plane area $A$ & $4 \times 4 \mathrm{~km}^{2}$ \\
Spectrum allocation (UL/DL) & $20 \mathrm{MHz}$ \\
Duplex mode & Half duplex \\
Thermal noise power density & $-174 \mathrm{dBm} / \mathrm{Hz}$ \\
Matérn cluster radius $R$ & $12.5 \mathrm{~m}$ \\
Modified Thomas cluster standard variance $\sigma$ & $R / 3$ \\
Average Number of DUE pairs per cluster $m$ & 3 \\
Maximum DUE transmit power $P_{\max }$ & $23 \mathrm{dBm}$ \\
Minimum DUE transmit power $P_{\min }$ & $-40 \mathrm{dBm}$ \\
Coding gain & $0 \mathrm{~dB}$ \\
DUE antenna gain & $0 \mathrm{dBi}$ \\
DUE noise figure & $9 \mathrm{~dB}$ \\
DUE link spectrum efficiency & $0.05 \mathrm{bit} / \mathrm{s} / \mathrm{Hz}$ \\
Carrier frequency & $2.4 \mathrm{GHz}$ \\
Path loss exponent & 2.7 \\
Common transmit power of DUE $P_{d}$ & $-15 \mathrm{dBm}$ \\
Simulation iterations & $5 \times 10^{4}$ \\
\hline
\end{tabular}

interior-point algorithm is used in the simulations. During each iteration, one cluster closest to the plane center is selected as the target cluster. The benchmarks in the simulations are conventional open loop and fixed power schemes [46], [51]. The DUE transmit power for the physical uplink shared channel (PUSCH) transmission is given by [51]

$$
P_{P U S C H}^{i j}=\min \left\{P_{\max }, 10 \log _{10} M+P_{0}+a l_{i j}\right\}[\mathrm{dBm}],
$$

where $M$ is the number of assigned RBs; $P_{0}=\{\beta+$ $\left.P_{N}\right\}[\mathrm{dBm} / \mathrm{RB}], \beta$ is the open loop target signal to noise ratio (SNR), $P_{N}$ is the noise power per $\mathrm{RB}$, and $l_{i j}=$ $-10 \log _{10}\left(G_{e} \ell\left(d_{i j}\right)\right)$; $a$ is the cell-specific path loss compensation factor. We assume that all $\mathrm{RBs}$ are allocated to each DUE and adopt conventional settings as the open loop scheme with $a=1$ (i.e., full path loss inversion). For the optimization of the target cluster, we assumed that the transmit power of all DUE transmitters in interfering clusters before the optimization is given by $P_{d}$.

When maximizing the joint coverage probability for the target cluster/whole network, the optimization was performed first in the target/all cluster(s), and the corresponding averaged transmit power was achieved for the target/all cluster(s). Then, the fixed power scheme was performed in the target/all cluster(s) using the average of previously optimized power levels in the target/all cluster(s). Finally, the open loop is performed in the target/all cluster(s). For the target cluster optimization, since the transmit power of all cluster before optimization is $P_{d}$ and the optimized power within the target cluster is upper bounded by $P_{d}$, the optimization would not increase the joint coverage probability of the target cluster in trade of degrading the coverage probabilities of neighboring clusters.

When minimizing power consumption for the target cluster/whole network, the fixed power scheme with power level $P_{d}$ was performed first in the target/all cluster(s), and the corresponding joint coverage probability of the target/all cluster(s) was achieved. Then, the optimization scheme was performed in the target/all cluster(s) with the threshold $\bar{p}_{s}$ given by the joint coverage probability of the target/all cluster(s) from the fixed power scheme. For the optimizations based on noncoherent lower bound and single-cluster approximation, if there is no feasible solution found, the transmit power of the DUEs will be given by $P_{d}$. The simulation results are obtained by averaging over 50000 independent Monte Carlo trials. 


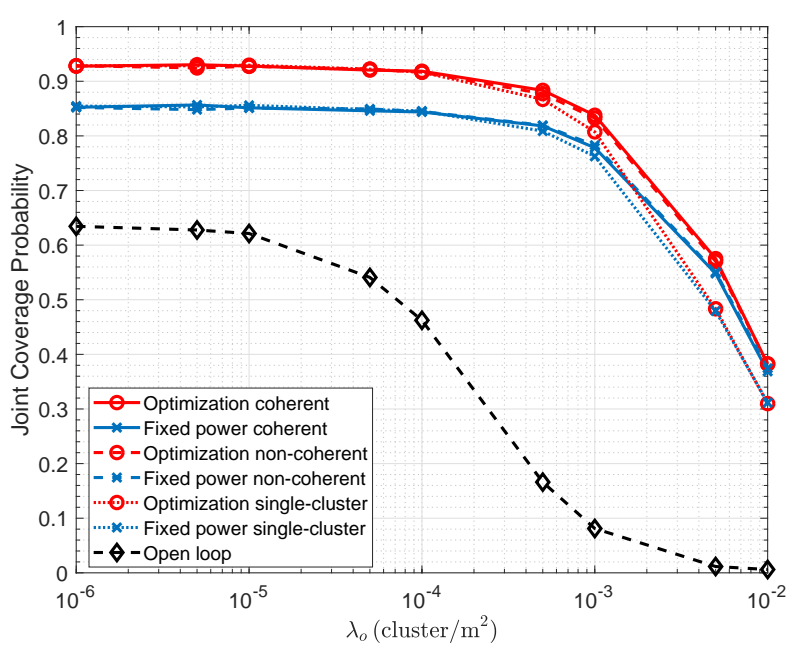

(a) Matérn.

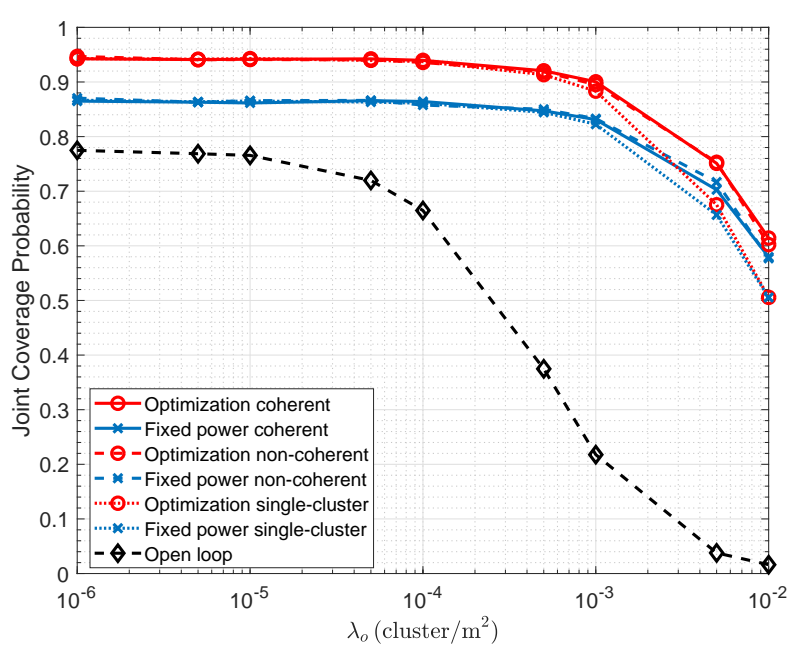

(b) Modified Thomas.

Fig. 2: Joint coverage probability of the target cluster vs. $\lambda_{o}$ when using joint coverage probability maximization, open loop and fixed power schemes.

\section{B. Simulation results}

1) Maximizing joint coverage probability of the target cluster: Fig. 2 gives the joint coverage probabilities of the DUEs in the target cluster after applying different power allocation schemes. The plots of all the simulation results on joint coverage probabilities are based on the coherent coverage probability formulation. One can easily see that all performance curves drop heavily as the cluster intensity $\lambda_{o}$ increases. The distribution of the DUEs within clusters has a noticeable effect on the performance given the same $\bar{m}$. Among the optimizations, the performance of the optimization based on the coherent formulation is the best. The optimization based on the non-coherent lower bound has slightly worse performance than the coherent formulation, while the singlecluster approximation based optimization performs the worst. Compared with other schemes, the open loop scheme performs the worst. This is because it only compensates for the path

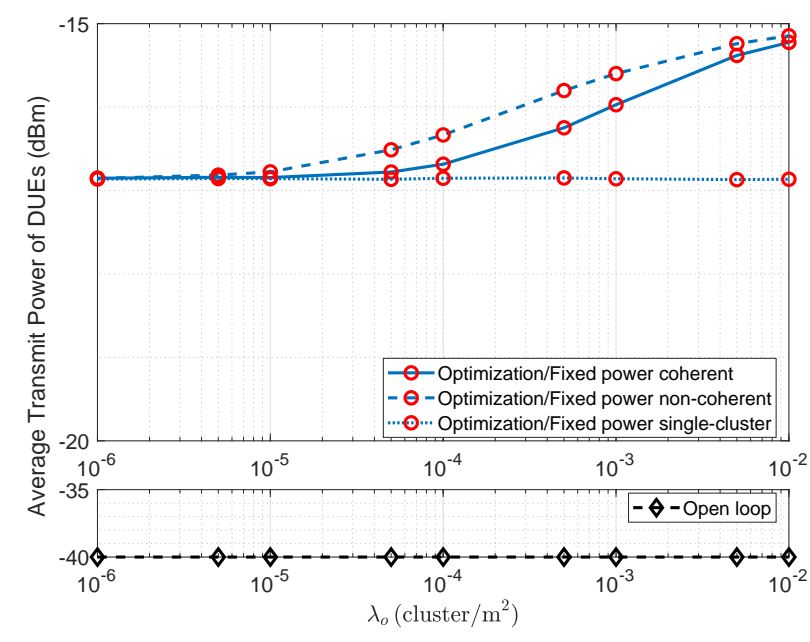

(a) Matérn.

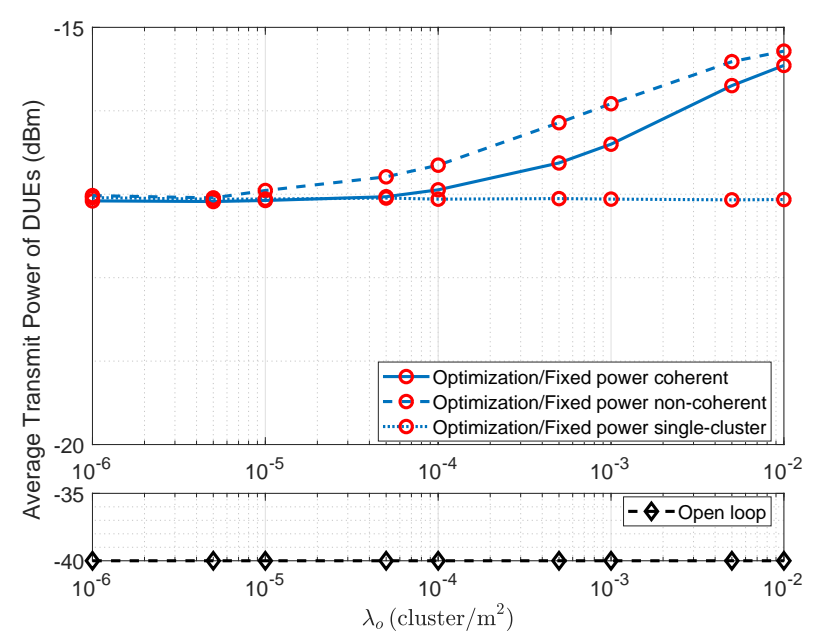

(b) Modified Thomas.

Fig. 3: Average transmit power of the DUE in the target cluster vs. $\lambda_{o}$ when using joint coverage probability maximization, open loop and fixed power schemes.

loss from the transmitter to the intended receiver without considering the fading and interference. Thus, as the cluster intensity increases, open loop would not be suitable for out-ofband D2D networks. Meanwhile, all the optimization schemes perform better than their fixed power counterparts which use the averaged power of the target cluster after the optimization over each realization. In details, when the cluster intensity is below $10^{-4}$ cluster $/ \mathrm{m}^{2}$, the proposed optimizations have $8 \%$ better joint coverage probabilities than their fixed power counterparts. Besides, all the optimizations have the same performance in the low intensity. This is because the intercluster interference is negligible at lower densities. Hence, the joint coverage probability based on the coherent formulation, non-coherent lower bound and single-cluster approximation are approximately the same. Furthermore, compared with other proposed schemes at higher cluster intensity, the optimization based on the single-cluster approximation performs the worst 


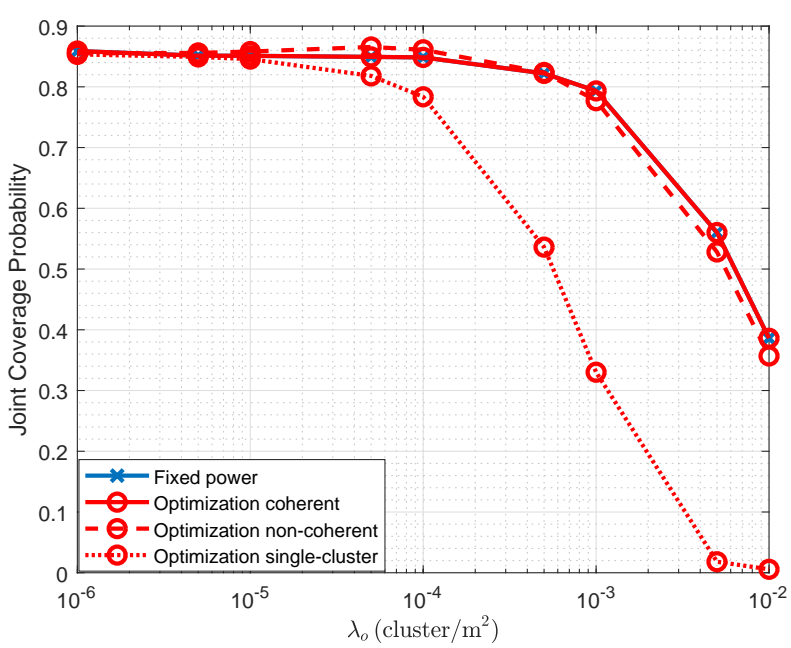

(a) Matérn.

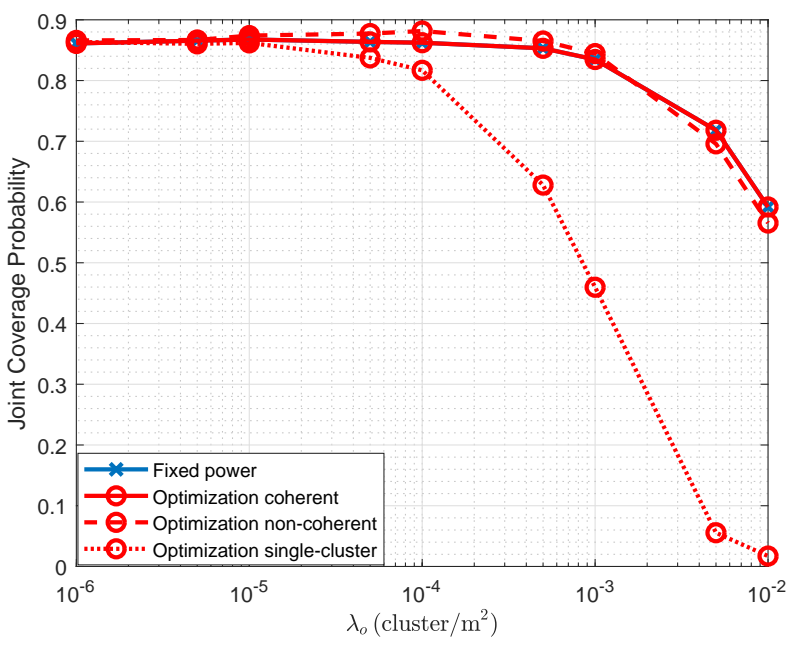

(b) Modified Thomas.

Fig. 4: Joint coverage probability of the target cluster vs. $\lambda_{o}$ given by benchmarking the power consumption minimization against the fixed power scheme.

since it ignores the inter-cluster interference from neighboring clusters.

Fig. 3 compares the average power consumption of the typical DUE in the target cluster with different power allocation schemes. Both the Matérn cluster and modified Thomas cluster processes have the similar trend in the average power consumption for different cluster intensities. In the proposed scheme, the optimizations based on the coherent formulation and single-cluster approximation consume the least amount of power. The average transmit power levels from the optimization based on the single-cluster approximation are the same for different cluster intensities since the single-cluster approximation only considers the DUEs within the target cluster and $\bar{m}$ is fixed. Meanwhile, the open loop scheme achieves the lowest power consumption at $-40 \mathrm{~dB}$ since it only compensates for the path loss among the DUEs. According to (29), when the distance is too short, the minimum

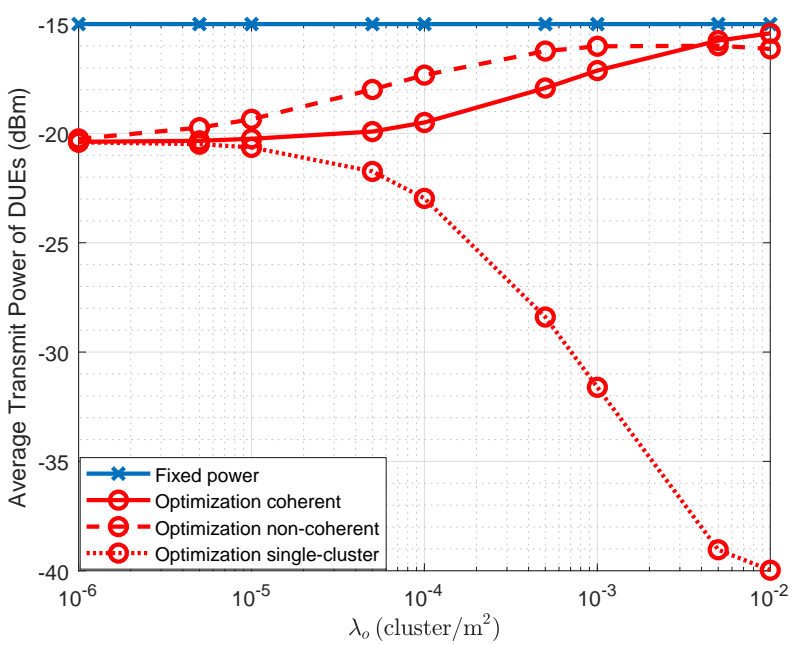

(a) Matérn.

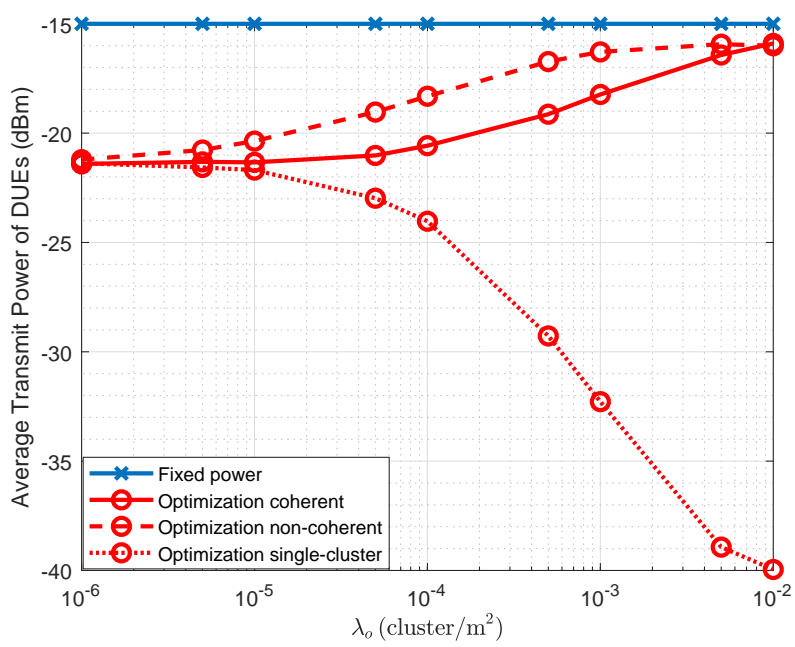

(b) Modified Thomas.

Fig. 5: Transmit power of the typical DUE in the target cluster vs. $\lambda_{o}$ given by benchmarking the power consumption minimization against the fixed power scheme.

transmit power is given. Compared with the upper limit of the transmit power at $-15 \mathrm{dBm}$, the optimizations based on the coherent and non-coherent lower bound formulations save the transmit power by at most $2 \mathrm{~dB}$ at $\lambda_{o}=10^{-6}$ cluster $/ \mathrm{m}^{2}$ and $0.45 \mathrm{~dB}$ at $\lambda_{o}=10^{-2}$ cluster $/ \mathrm{m}^{2}$. The reason why there is almost no power saving in the high cluster intensity region can be explained as follows. As $\lambda_{o}$ increases, the inter-cluster interference becomes dominant, and the proposed optimizations based on both coherent and non-coherent lower bound formulations must boost the transmit power of the DUEs within the target cluster to increase the SINR, which ends up with reaching the upper bound of transmit power.

2) Minimizing power consumption of the target cluster: Fig. 4 depicts the achieved joint coverage probabilities of the target cluster for power minimization using the fixed power scheme as the benchmark. In the fixed power scheme, the transmitter power for all clusters is given by $P_{d}$. During each 
trial, the threshold $\bar{p}_{s}$ is given by the joint coverage probability from the fixed power scheme over the same realization of the clusters. The joint coverage probabilities in the modified Thomas cluster process is slightly better than the Matérn cluster process. The open loop scheme is omitted in the simulation since there are no parameters allowed to adjust so that it could reach a predefined threshold for the joint coverage probability. Meanwhile, it is evident that the optimization based on the coherent formulation gives a close match to the threshold $\bar{p}_{s}$. This can be explained by the fact that when minimizing power consumption, the coherent formulation has full knowledge of all the node's locations and transmit powers. Hence, it can guarantee that the threshold value is achieved. The optimization based on the non-coherent lower bound formulation performs better than $\bar{p}_{s}$ when $\lambda_{o}$ is less than $10^{-3}$ cluster $/ \mathrm{m}^{2}$ while achieving the worse performance when $\lambda_{o}$ is greater than $10^{-3}$ cluster $/ \mathrm{m}^{2}$. The optimization based on the single-cluster approximation is far from the threshold as $\lambda_{o}$ is increasing.

Fig. 5 shows the corresponding average transmit power of DUEs as discussed in Fig. 4. Among all optimization formulations, the joint coverage probability based on the single-cluster approximation achieves the minimum power consumption, but the corresponding joint coverage probabilities are far away from achieving the required $\bar{p}_{s}$. Compared with the fixed power scheme, we see that the optimization based on the coherent formulation saves power by at most $1 \mathrm{~dB}$ to $6 \mathrm{~dB}$ from $\lambda_{o}=10^{-6}$ to $\lambda_{o}=10^{-2}$, while there is a maximum gap of $2 \mathrm{~dB}$ between it and the non-coherent formulation at some intensities such as $\lambda_{o}=10^{-4}$. The performance drop slows down from $\lambda_{o}=5 \times 10^{-3}$ cluster $/ \mathrm{m}^{2}$ to $\lambda_{o}=10^{-2}$ cluster $/ \mathrm{m}^{2}$ is due to the optimization has reach the lower bound of the transmit power at $\lambda_{o}=10^{-2}$ cluster $/ \mathrm{m}^{2}$. As a result of these observations, the coherent and non-coherent formulations are more suitable for the idea of green communications, while giving similar performance to that of the fixed power scheme.

3) Maximizing joint coverage probability of the whole network: As shown in Fig. 6(a), the joint coverage probability of the whole network increases as $A$ decreases, where an average of 16 Matérn clusters are within the network ${ }^{3}$. Compared with the full power and open loop schemes, the proposed optimization performs the best. The fixed power schemes have a similar joint coverage probability to their optimization counterparts when $A$ is below $10^{2} \mathrm{~m}^{2}$ and around $10 \%$ smaller when $A$ is above $10^{4} \mathrm{~m}^{2}$. Meanwhile, Fig. 6(b) shows that the proposed optimizations consume around $2 \mathrm{~dB}$ less power than the upper transmit power limit $\mathrm{P}_{\mathrm{U}}=-15 \mathrm{dBm}$ when $A$ is above $10^{4} \mathrm{~m}^{2}$. When $A$ is below $10^{4} \mathrm{~m}^{2}$, there is still power saving, but the saving drops as $A$ decreases. Furthermore, the optimizations based on both the coherent

\footnotetext{
${ }^{3}$ Due to the similar trending in achieving the joint coverage probability and average power consumption for both the Matérn cluster and modified Thomas cluster processes, only the discussion on the Matérn cluster processes is presented in this subsection. Meanwhile, different from previous settings for the target cluster optimizations which changes $\lambda_{o}$ under a fixed $A$, the simulations for all cluster optimizations changes $A$ for an average of 16 Matérn clusters within $A$. This is because as the number of clusters increases, the optimization speed decreases heavily and it will take a very long time to complete $5 \times 10^{4}$ trials.
}

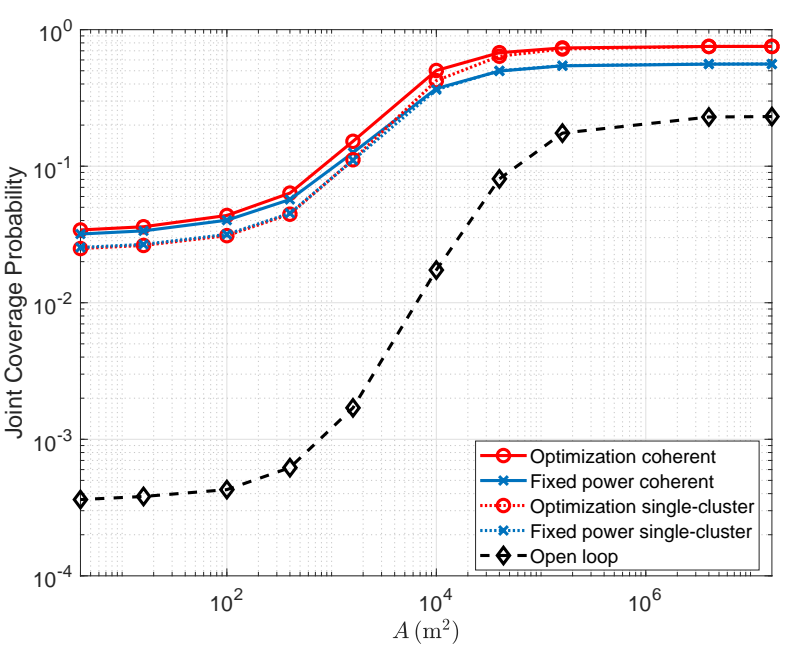

(a)

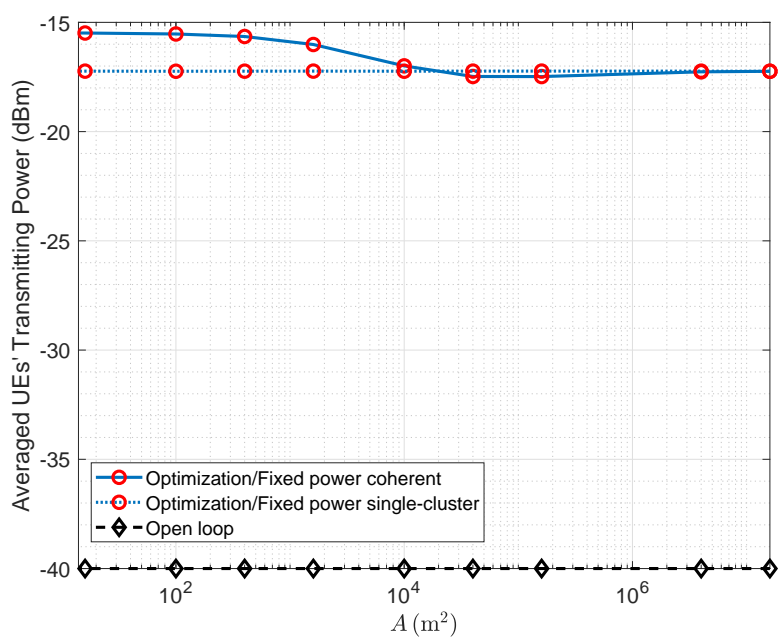

(b)

Fig. 6: The joint coverage probability (a) and average transmit power (b) of all clusters vs. $A$ given by joint coverage probability maximization, open loop and fixed power schemes.

formulation and single-cluster approximation spend the same average transmit power and achieve the same joint coverage probability when $A$ is large. This is because only the intracluster interference is taking effect at a large $A$, and both the coherent formulation and single-cluster approximation have captured this interference in their formulations. When $A$ is getting smaller, the inter-cluster interference becomes nonnegligible, and just the optimization based on the coherent formulation could reduce the interference further at the cost of increasing the average transmit power of the DUEs within the network.

4) Minimizing power consumption of the whole network: Fig. 7 gives the joint coverage probability of the whole network and the average transmit power after power consumption minimization given the fixed power scheme as the benchmark. As shown in Fig. 7(a), the coherent optimization achieves similar performance to the fixed power scheme when 


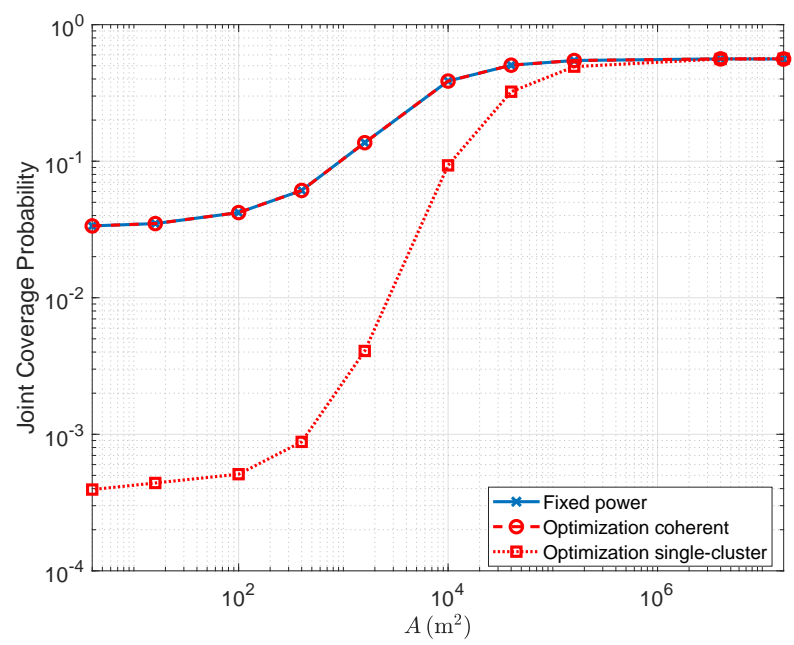

(a)

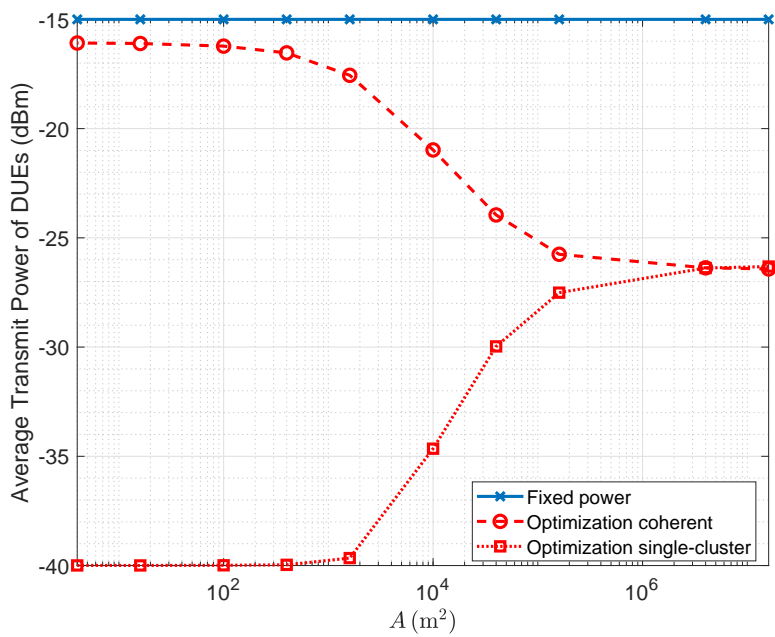

(b)

Fig. 7: The joint coverage probability (a) and average transmit power (b) of all clusters vs. $A$ given by benchmarking the power consumption minimization against the fixed power scheme.

the threshold $\bar{p}_{s}$ during each simulation trial is obtained by the joint coverage probability of the fixed power scheme. Meanwhile, the optimization based on the single-cluster approximation fails to achieve the required $\bar{p}_{s}$ when $A$ is small. That is because, the inter-cluster interference increases as $A$ decreases for a given average number of clusters in $A$, and the single-cluster approximation does not consider the inter-cluster interference. The average transmit power in Fig. 7(b) shows that the optimization based on the coherent formulation saves around $1 \mathrm{~dB}$ to $11 \mathrm{~dB}$ for the given range of $A$. Hence, the optimization based on the coherent formulation could be applied to power saving while maintaining the same performance as the fixed power scheme. Meanwhile, the optimization based on the single-cluster approximation has a similar performance to the optimization based on the coherent formulation when $A$ is very large. That is because, when $A$ is very large, only the intra-cluster interference taking effect, and both formulations have taken the intra-cluster interference into account.

\section{CONClusion}

In this paper, we optimized the performance of an out-ofband D2D network characterized by joint coverage probability maximization and power minimization. The joint coverage probabilities formulated based on the coherent, non-coherent lower bound and single-cluster approximation coverage probabilities make the optimizations feasible to both centralized and localized communication networks. Compared with conventional open loop and fixed power allocation schemes, the joint coverage probability of the target cluster is maximized with even smaller power consumption. The power minimization enables DUEs to have optimal total power consumption while maintains the same performance compared with the fixed power scheme. These optimization frameworks will facilitate the applications of commercial and public safety and fulfill the idea of green D2D communications in $5 \mathrm{G}$ communication networks and beyond.

\section{APPENDIX}

\section{A. Proof of Lemma 1}

For the Matérn cluster process,

$$
\begin{aligned}
& \mathcal{L}_{I}(s)=\exp \left(-2 \pi \lambda_{o} \int_{0}^{\infty}\left[1-\xi\left(s, R_{y}\right)\right] R_{y} \mathrm{~d} R_{y}\right) \\
& \stackrel{\text { (a) }}{\geq} \exp \left(-2 \pi \lambda_{o} \int_{0}^{\infty} \int_{0}^{\infty} \frac{\bar{m} g(x)}{s^{-1}+g(x)} f_{R_{d}}\left(x \mid R_{y}\right) \mathrm{d} x R_{y} \mathrm{~d} R_{y}\right) \\
& \stackrel{\text { (b) }}{=} \exp \left(-2 \pi \lambda_{o} \bar{m} \int_{0}^{\infty} \frac{g(x)}{s^{-1}+g(x)} x \mathrm{~d} x\right) \\
& =\exp \left(-\pi \lambda_{o} \bar{m} \frac{\left(s G_{e}\left(\frac{\lambda}{4 \pi}\right)^{2} P_{d}\right)^{2 / \alpha}}{\operatorname{sinc}(2 / \alpha)}\right)
\end{aligned}
$$

where (a) is the result of $\exp (-a x) \geq 1-a x$ for $a \geq 0$; (b) is because $\int_{0}^{\infty} f_{R_{d}}\left(x \mid R_{y}\right) R_{y} \mathrm{~d} R_{y}=\int_{0}^{\infty} x f_{R_{d}}\left(R_{y} \mid x\right) \mathrm{d} R_{y}=$ $x$, which is given by Lemma 2 . This concludes our proof.

Lemma 2. $\int_{0}^{\infty} f_{R_{d}}\left(x \mid R_{y}\right) R_{y} \mathrm{~d} R_{y}=\int_{0}^{\infty} x f_{R_{d}}\left(R_{y} \mid\right.$ $x) \mathrm{d} R_{y}=x$, where $f_{R_{d}}\left(x \mid R_{y}\right)$ is given by (10).

Proof: For a given $x \geq 0$, (10) guarantees that $x$ and $R_{y}$ are interchangeable in terms of both equations and domains in $f_{R_{d}}\left(x \mid R_{y}\right) R_{y}$. Thus, $\int_{0}^{\infty} f_{R_{d}}\left(x \mid R_{y}\right) R_{y} \mathrm{~d} R_{y}=$ $\int_{0}^{\infty} x f_{R_{d}}\left(R_{y} \mid x\right) \mathrm{d} R_{y}$. Meanwhile, for a given $x, f_{R_{d}}\left(R_{y} \mid\right.$ $x)$ is a marginal probability density function. Therefore, $\int_{0}^{\infty} x f_{R_{d}}\left(R_{y} \mid x\right) \mathrm{d} R_{y}=x \int_{0}^{\infty} f_{R_{d}}\left(R_{y} \mid x\right) \mathrm{d} R_{y}=x$. This concludes our proof.

\section{B. Proof of Theorem 1}

The coherent and single-cluster approximated coverage probability formulations have similar mathematical structures to the one in [52], within which the proof of Theorem 1 is given. The proof of non-coherent lower-bound coverage probability formulation is given as follows:

For a non-coherent coverage probability between the transmitting node $i$ and receiving node $j$ given by (17), applying 
variable transformations $\tilde{P}_{i}=\ln P_{i}$ and taking the natural logrithm on both sides of the equation, we have $\ln p_{i j}^{n c}=$ $\ln p_{i j}^{s c}+\ln \mathcal{L}_{I}\left(\frac{\beta}{G_{e} \ell\left(d_{i j}\right) \exp \left(\tilde{P}_{i}\right)}\right) \cdot \ln p_{i j}^{s c}$ is concave in $\tilde{\mathbf{P}}_{\kappa}[52]$ and $\ln \mathcal{L}_{I}\left(\frac{\beta}{G_{e} \ell\left(d_{i j}\right) \exp \left(\tilde{P}_{i}\right)}\right) \geq-\pi \lambda_{o} \bar{m} \frac{\left(\beta d_{i j}^{\alpha} \exp \left(-\tilde{P}_{i}\right) P_{d}\right)^{2 / \alpha}}{\operatorname{sinc}(2 / \alpha)}$, where the right side of the inequality is also concave in $\tilde{P}_{i} \in$ $\tilde{\mathbf{P}}_{\kappa}$, since its second order derivative with respect to $\tilde{P}_{i}$ equals $-A\left(\frac{2}{\alpha}\right)^{2} \exp \left(-\frac{2}{\alpha} \tilde{P}_{i}\right)<0$, where $A=\pi \lambda_{o} \bar{m} \frac{\left(\beta d_{i j}^{\alpha} P_{d}\right)^{2 / \alpha}}{\operatorname{sinc}(2 / \alpha)}$. Therefore, the lower bound on $p_{i j}^{n c}$ given by (15) is logconcave in $\tilde{\mathbf{P}}_{\kappa}$. The objective in (19) is also log-concave, because the product of log-concave coverage probabilities is still log-concave [52]. This concludes our proof.

\section{Proof of Theorem 2}

Let $\tilde{P}_{i}=\ln P_{i}$ and take the natural logarithm on (23) (25), we have a transformed optimization problem as (26) (28). A function with a log-sum-exponent form given by (26) is convex in $\tilde{\mathbf{P}}_{\kappa}$ [47, pp. 72 and 74]. In (28), the function $\ln \bar{p}_{s}-\ln p_{s}\left(\tilde{\mathbf{P}}_{\kappa}\right)$ is convex in $\tilde{\mathbf{P}}_{\kappa}$, because: 1) according to Theorem $1,-\ln p_{s}\left(\tilde{\mathbf{P}}_{\kappa}\right)$ is convex for the coherent, noncoherent lower bound and single-cluster approximated coverage probability formulations; 2) the affine operation that adding $\ln \bar{p}_{s}$ to $-\ln p_{s}\left(\tilde{\mathbf{P}}_{\kappa}\right)$ keeps the convexity in the result [47]. Therefore, the optimization problem given by (26) - (28) is convex in $\tilde{\mathbf{P}}_{\kappa}$. This concludes our proof.

\section{ACKNOWLEDGMENT}

The authors would like to thank Mr. Shuping Dang who assisted in the proofreading of the manuscript.

\section{REFERENCES}

[1] D. Tsolkas, E. Liotou, N. Passas, and L. Merakos, "LTE-A access, core, and protocol architecture for D2D communication," in Smart Device to Smart Device Commun. Springer, 2014, pp. 23-40.

[2] S. Dang, G. Chen, and J. P. Coon, "Outage performance analysis of full-duplex relay-assisted device-to-device systems in uplink cellular networks," IEEE Trans. Veh. Technol., Sept. 2016.

[3] Y. Wu, W. Guo, H. Yuan, L. Li, S. Wang, X. Chu, and J. Zhang, "Deviceto-device meets LTE-Unlicensed," IEEE Commun. Mag., vol. 54, no. 5, pp. 154-159, 2016.

[4] D. Câmara and N. Nikaein, Wireless Public Safety Networks Volume 1: Overview and Challenges. London,UK: ISTE Press Ltd, 2015.

[5] A. Asadi, Q. Wang, and V. Mancuso, "A survey on device-to-device communication in cellular networks," IEEE Commun. Surveys Tuts., vol. 16, no. 4, pp. 1801-1819, Apr. 2014.

[6] S. Dang, J. P. Coon, and G. Chen, "Resource allocation for full-duplex relay-assisted device-to-device multicarrier systems," IEEE Trans. Wireless Commun. Lett., vol. 6, no. 2, pp. 166-169, Jan. 2017.

[7] M. Usman, A. A. Gebremariam, U. Raza, and F. Granelli, "A softwaredefined device-to-device communication architecture for public safety applications in 5G networks," IEEE Access, vol. 3, pp. 1649-1654, Sept. 2015.

[8] C.-F. Chiasserini, "Content wanted: A different shade of D2D communications," in Proc. 2015 Int. Conf. Comput. Netw. Commun. (ICNC). Anaheim, CA, USA: IEEE, Feb. 2015, pp. 562-566.

[9] S. Han, H. Lee, J. Kim, and W. Lee, "On the connectivity in opportunistic D2D networks with hierarchical and non-hierarchical clustering," in Proc. IEEE Globecom Workshops (GC Wkshps). Washington, D.C., USA: IEEE, Dec. 2016.

[10] ITU, "IMT Vision - Framework and overall objectives of the future development of IMT for 2020 and beyond," ITU, Recommendation M.2083-0, Sept. 2015. [Online]. Available: https://www.itu.int/rec/RREC-M.2083-0-201509-I/en (Accessed Sept. 15, 2018)
[11] A. Asadi and V. Mancuso, "Network-assisted outband D2D-clustering in 5G cellular networks: theory and practice," IEEE Trans. Mobile Comput., vol. 16, no. 8, pp. 2246-2259, Oct. 2017.

[12] A. Al-Hourani, S. Kandeepan, and A. Jamalipour, "Stochastic geometry study on device-to-device communication as a disaster relief solution," IEEE Trans. Veh. Technol., vol. 65, no. 5, pp. 3005-3017, May 2016.

[13] R. K. Ganti and M. Haenggi, "Interference and outage in clustered wireless ad hoc networks," IEEE Trans. Inf. Theory, vol. 55, no. 9, pp. 4067-4086, Sept. 2009.

[14] Y. Zhang, L. Song, W. Saad, Z. Dawy, and Z. Han, "Contract-based incentive mechanisms for device-to-device communications in cellular networks," IEEE J. Sel. Areas Commun., vol. 33, no. 10, pp. 2144-2155, Oct. 2015

[15] G. George, R. K. Mungara, and A. Lozano, "An analytical framework for device-to-device communication in cellular networks," IEEE Trans. Wireless Commun., vol. 14, no. 11, pp. 6297-6310, Nov. 2015.

[16] M. Afshang and H. S. Dhillon, "Spatial modeling of device-to-device networks: Poisson cluster process meets Poisson hole process," in Proc. ASILOMAR, Pacific Grove, CA, USA, 2015.

[17] C. Wu, T. Yoshinaga, X. Chen, L. Zhang, and Y. Ji, "Cluster-based content distribution integrating lte and ieee $802.11 \mathrm{p}$ with fuzzy logic and q-learning," IEEE Comput. Intell. Mag., vol. 13, no. 1, pp. 41-50, Feb. 2018.

[18] Z. Jakó and G. Jeney, "Outage probability in Poisson-cluster-based LTE two-tier femtocell networks," Wireless Commun. Mob. Comput., vol. 15, no. 18, pp. 2179-2190, June 2015.

[19] M. Afshang, H. Dhillon, and P. Chong, "Modeling and performance analysis of clustered device-to-device networks," IEEE Trans. Wireless Commun., vol. 15, no. 7, pp. 4957-4972, July 2016.

[20] S. Krishnan and H. S. Dhillon, "Exact characterization of spatiotemporal joint coverage probability in cellular networks," in Proc. IEEE WCNC, San Francisco, CA, USA, March 2017.

[21] S. Mukherjee, Coverage analysis using the Poisson point process model. Cambridge, UK: Cambridge Uni. Press, 2013, pp. 44-81.

[22] Krishnan, Shankar and Dhillon, Harpreet S, "Spatio-temporal interference correlation and joint coverage in cellular networks," IEEE Trans. Wireless Commun., vol. 16, no. 9, pp. 5659-5672, June 2017.

[23] M. A. Kishk and H. S. Dhillon, "Joint uplink and downlink coverage analysis of cellular-based RF-powered IoT network," IEEE Trans. Green Commun. Netw., vol. 2, no. 2, pp. 446-459, June 2018.

[24] M. Noura and R. Nordin, "A survey on interference management for device-to-device (D2D) communication and its challenges in 5G networks," J. Netw. Comput. Appl., vol. 71, pp. 130-150, Aug. 2016.

[25] H. ElSawy, E. Hossain, and M.-S. Alouini, "Analytical modeling of mode selection and power control for underlay D2D communication in cellular networks," IEEE Trans. Commun., vol. 62, no. 11, pp. 41474161, Nov. 2014.

[26] R. Cai, J.-K. Zhang, T. N. Davidson, W. Zhang, K. M. Wong, and P.C. Ching, "A power allocation strategy for multiple Poisson spectrumsharing networks," IEEE Trans. Wireless Commun., vol. 14, no. 4, pp. 1785-1799, Apr. 2015.

[27] A. H. Sakr and E. Hossain, "Cognitive and energy harvesting-based D2D communication in cellular networks: Stochastic geometry modeling and analysis," IEEE Trans. Commun., vol. 63, no. 5, pp. 1867-1880, May 2015.

[28] N. Lee, X. Lin, J. G. Andrews, and R. W. Heath, "Power control for D2D underlaid cellular networks: Modeling, algorithms, and analysis," IEEE J. Sel. Areas Commun., vol. 33, no. 1, pp. 1-13, Jan. 2015.

[29] S. Panichpapiboon, G. Ferrari, and O. K. Tonguz, "Optimal common transmit power in ad hoc wireless networks," in Proc. 24th Int. Performance Comput. Commun. Conf. (IPCCC). Phoenix, AZ, USA: IEEE, 2005, pp. 593-597.

[30] T. Ozan, K. and F. Gianluigi, Eds., Ad Hoc Wireless Networks: A Communication-Theoretic Perspective. Chichester, West Sussex, England: John Wiley \& Sons Ltd, 2006.

[31] S. Panichpapiboon, G. Ferrari, and O. K. Tonguz, "Optimal transmit power in wireless sensor networks," IEEE Trans. Mobile Comput., vol. 5, no. 10, pp. 1432-1447, Oct. 2006.

[32] E. Hossain, V. K. Bhargava, and G. P. Fettweis, Green Radio Communication Networks. Cambridge, UK: Cambridge Uni. Press, 2012.

[33] A. Baddeley, E. Rubak, and R. Turner, Spatial point patterns: methodology and applications with $R$. Boca Raton, FL, USA: CRC Press, 2015.

[34] Z. Wu, V. D. Park, and J. Li, "Enabling device to device broadcast for LTE cellular networks," IEEE J. Sel. Areas Commun., vol. 34, no. 1, pp. 58-70, Jan. 2016. 
[35] M. Afshang, H. S. Dhillon, and P. H. J. Chong, "Fundamentals of clustercentric content placement in cache-enabled device-to-device networks," IEEE Trans. Commun., vol. 64, no. 6, pp. 2511-2526, June 2015.

[36] M. I. Ribeiro, "Gaussian probability density functions: Properties and error characterization,” Instituto Superior Tecnico, Lisboa, Portugal, Tech. Rep., Feb. 2004.

[37] S. Guo, F. Wang, Y. Yang, and B. Xiao, "Energy-efficient cooperative transmission for simultaneous wireless information and power transfer in clustered wireless sensor networks," IEEE Trans. Commun., vol. 63 , no. 11, pp. 4405-4417, Nov. 2015.

[38] E. McCune, Practical digital wireless signals, ser. The Cambridge RF and Microwave Engineering Series. Cambridge, UK: Cambridge Uni. press, 2013.

[39] S. Weber, J. G. Andrews, and N. Jindal, "The effect of fading, channel inversion, and threshold scheduling on ad hoc networks," IEEE Trans. Inf. Theory, vol. 53, no. 11, pp. 4127-4149, Nov. 2007.

[40] C.-H. Liu, B. Rong, and S. Cui, "Optimal discrete power control in Poisson-clustered ad hoc networks," IEEE Trans. Wireless Commun., vol. 14, no. 1, pp. 138-151, Jan. 2015.

[41] J. G. Andrews, F. Baccelli, and R. K. Ganti, "A tractable approach to coverage and rate in cellular networks," IEEE Trans. Commun., vol. 59, no. 11 , pp. 3122-3134, Nov. 2011

[42] G. Chen and J. A. Chambers, "Exact outage probability analysis for cooperative AF relay network with relay selection in presence of intercell interference," Electron. Lett., vol. 48, no. 21, pp. 1346-1347, Oct. 2012.

[43] G. Chen, Y. Gong, P. Xiao, and R. Tafazolli, "Dual antenna selection in self-backhauling multiple small cell networks," IEEE Commun. Lett., vol. 20, no. 8, pp. 1611-1614, Aug. 2016.

[44] J. Tang, G. Chen, J. P. Coon, and D. E. Simmons, "Distance distributions for Matérn cluster processes with application to network performance analysis," in Proc. IEEE ICC, Paris, France, May 2017.

[45] 3GPP, "FDD UE minimum transmission power," 3GPP, Tech. Rep. 8(99737), Oct. 1999. [Online]. Available: http://www.3gpp.org/ftp/tsg_ran/wg4_radio/TSGR4_08/Docs/PDFs/r499737.pdf (Accessed Sept. 15, 2018)

[46] 3GPP, "Evolved Universal Terrestrial Radio Access (EUTRA); User Equipment (UE) radio transmission and reception," 3GPP, TS 36.101, Jan. 2016. [Online]. Available: http://www.3gpp.org/dynareport/36101.htm (Accessed Sept. 15, 2018)

[47] S. Boyd and L. Vandenberghe, Convex optimization. Cambridge Uni. Press, 2004

[48] J. Mattingley and S. Boyd, "Real-time convex optimization in signal processing," IEEE Signal Process. Mag., vol. 27, no. 3, pp. 50-61, May 2010.

[49] R. Hauser. Interior-point methods for inequality constrained optimization. Oxford University. [Online]. Available: https://people.maths.ox.ac.uk/hauser/hauser_lecture4.pdf (Accessed Sept. 15, 2018)

[50] R. A. Waltz, J. L. Morales, J. Nocedal, and D. Orban, "An interior algorithm for nonlinear optimization that combines line search and trust region steps," Mathematical programming, vol. 107, no. 3, pp. 391-408, July 2006.

[51] F. Khan, LTE for $4 G$ mobile broadband: air interface technologies and performance. New York, NY, USA: Cambridge Uni. Press, 2009.

[52] J. Tang, J. P. Coon, and G. Chen, "Optimal cross-tier power allocation for D2D multi-cell networks," in Proc. IEEE GLOBECOM, Washington, D.C., USA, Dec. 2016.

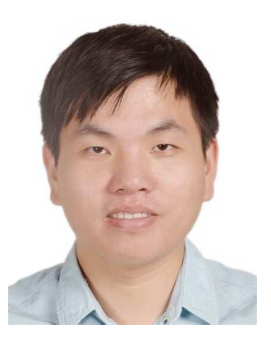

Jinchuan Tang (S'17) received his B.Eng. degree in electronic information engineering from Chongqing University of Posts and Telecommunications in 2011. He worked as an Assistant Engineer in Huawei Technologies Co., Ltd. from 2011 to 2012. He obtained the M.Sc. degree with Distinction in wireless communications from the University of Southampton in early 2014 . He is currently pursuing a DPhil degree under the supervision of Professor Justin P. Coon with the Department of Engineering Science, University of Oxford. His current research interests include optimum power allocation, random geometric networks, secure communication, and route selection in wireless networks.

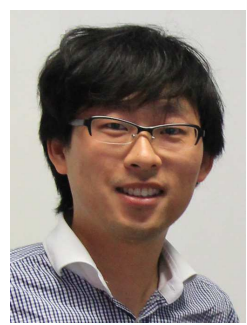

Gaojie Chen (S'09 - M'12) received the B. Eng. Degree in electrical information engineering and the B.Ec. Degree in international economics and trade from Northwest University, China, in 2006, and the M.Sc. (Hons.) and Ph.D. degrees in electrical and electronic engineering from Loughborough University, U.K., in 2008 and 2012, respectively. From 2008 to 2009, he was a Software Engineering with DTmobile, Beijing, China, and a Research Associate with the School of Electronic, Electrical and Systems Engineering, Loughborough University, from 2012 to 2013. Then he was a Research Fellow with the 5GIC, University of Surrey, U.K., from 2014 to 2015 . Then he was a Research Fellow at the University of Oxford, U.K., from 2015 to 2018 . He is currently a Lecturer at the University of Leicester, U.K. His current research interests include information theory, wireless communications, IoT, cognitive radio, secrecy communication and random geometric networks.

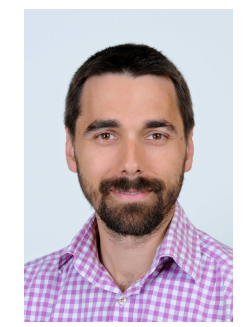

Justin P. Coon (S'02 - M'05 - SM'10) received the B.Sc. degree (Hons.) in electrical engineering from the Calhoun Honours College, Clemson University, USA, and the Ph.D. degree in communications from the University of Bristol, U.K., in 2000 and 2005, respectively. In 2004, he joined as a Research Engineer with the Bristol-based Telecommunications Research Laboratory (TRL), Toshiba Research Europe Ltd., where he was involved in research on a broad range of communication technologies and theories, including single- and multi-carrier modulation techniques, estimation and detection, diversity methods, and system performance analysis and networks. He held the research manager position from 2010 to 2013, during which time he led all theoretical and applied research on the physical layer at TRL. He was a Visiting Fellow with the School of Mathematics, University of Bristol, from 2010 to 2012, where he held a reader position with the Department of Electrical and Electronic Engineering from 2012 to 2013. He joined the University of Oxford in 2013, where he is currently an Associate Professor with the Department of Engineering Science and a Tutorial Fellow of Oriel College. He is the Technical Manager of the EU FP7 project DIWINE. He has authored in excess of 100 papers in leading international journals and conferences, and is a named inventor on over 30 patents. His research interests include communication theory, information theory, and network theory. Dr Coon was a recipient of TRLs Distinguished Research Award for his work on block-spread CDMA, aspects of which have been adopted as mandatory features in the 3GPP LTE Rel-8 standard. He was also a co-recipient of two best paper awards at the ISWCS 2013 and the EuCNC 2014. He received the award for Outstanding Contribution in 2014. $\mathrm{He}$ has served as an Editor for the IEEE TRANSACTIONS ON WIRELESS COMMUNICATIONS from 2007 to 2013, the IEEE TRANSACTIONS ON VEHICULAR TECHNOLOGY from 2013 to 2016 . He has been serving as an Editor for the IEEE WIRELESS COMMUNICATIONS LETTERS since 2016 and the IEEE COMMUNICATIONS LETTERS since 2017. 